\title{
ELECTROPHORETIC VARIANTS OF BLOOD PROTEINS IN JAPANESE
}

\section{CERULOPLASMIN}

\author{
Mikio FujtTa, Chiyoko Satoh, Jun-ichi Asakawa, \\ Yuko Nagahata, Yoshiko Tanaka, Ryuji Hazama, ${ }^{1}$ \\ and Kazuaki GoRIKI ${ }^{2}$ \\ Division of Biochemical Genetics, Department of Clinical Laboratories, \\ Radiation Effects Research Foundation, 5-2 Hijiyama Park, \\ Minami Ward, Hiroshima 730, Japan
}

\begin{abstract}
Summary The plasma ceruloplasmin of 22,367 children of A-bomb survivors in Hiroshima and Nagasaki was examined for variants by electrophoresis. The sample was composed of 14,964 unrelated children and 7,403 siblings of the unrelated persons. A total of seven types of electrophoretic variants were detected; four migrating anodally and three cathodally to the normal $\mathrm{B}$ band. We have reported two of these variants, $\mathrm{CP} \mathrm{A}_{\mathrm{NG1}}$ and $\mathrm{CP} \mathrm{C}_{\mathrm{NG1}}$, previously but the other five, CP $\mathrm{A}_{\mathrm{NG} 2}, \mathrm{CP} \mathrm{A}_{\mathrm{HR} 1}$, $\mathrm{CP} \mathrm{A}_{\mathrm{HR} 2}, \mathrm{CP} \mathrm{C}_{\mathrm{HR} 1}$, and $\mathrm{CP} \mathrm{C}_{\mathrm{HR} 2}$, are newly identified. The allelic frequency of $C P^{*} C N G 1$ was 0.00916 , so that the variant is considered to be a polymorphic allele. Homozygosity for the $C P^{*} C N G I$ allele was detected in five individuals. This is the first report of a homozygous phenotype for a ceruloplasmin variant in a Japanese population. Family study of the new five variants all demonstrated patterns of codominant inheritance.
\end{abstract}

\section{INTRODUCTION}

Ceruloplasmin (CP), first isolated from pig serum by Holmberg and Laurell in 1948 , is a copper-containing protein which electrophoretically comigrates with the $\alpha_{2}$-globulin fraction. McAlister et al. (1961) reported the first electrophoretic variant of $\mathrm{CP}$ in humans, a fast (more anodally) migrating variant termed $1 \mathrm{~F}$. Shreffler et al. (1967) then identified various types of CP variants in American Negroes and Caucasians and showed that these variants could be explained if three kinds of alleles, $C P^{*} A, C P^{*} B$, and $C P^{*} C$, associated respectively with a fast migrat-

Received May 13, 1985

Present address: ${ }^{1}$ Department of Medicine, Radiation Effects Research Foundation, 8-6, 1-chome, Nakagawa-cho, Nagasaki 850, Japan. ${ }^{2}$ Department of Internal Medicine, Mihara Medical Association Hospital, 370-1, Miyaura-cho, Mihara, Hiroshima 723, Japan. 
ing, the normal, and a slow migrating phenotype, are presumed to exist. Subsequently, Shokeir and Shreffler (1970), Tanis et al. (1973), Neel et al. (1977), and Mohrenwiser and Decker (1982) identified new variants. To date, nine types of variants which migrate anodally to normal $\mathrm{CP} B$, namely, CP Michigan, CP AMAC-1, CP A-YAN-1, CP A, CP A-CAY-1, CP A-WAP-1, CP Th, CP Bpt, and $\mathrm{CP} \mathrm{A}_{\mathrm{NG} 1}$, and six which migrate cathodal to normal $\mathrm{CP} B$, namely, $\mathrm{CP} \mathrm{NH}, \mathrm{CP}$ CAnnArbor-1, CP C-WAP-1, CP C, CP C-WAP-2, and $\mathrm{CP} \mathrm{C}_{\mathrm{NG} 1}$, have been reported. Four of these alleles achieve polymorphic proportions, $C P^{*} A$ in Negroid, Caucasoid and Indians (Shreffler et al., 1967; Kellermann and Walter, 1972; Bajatzadeh and Walter, 1969; Mohrenweiser and Decker, 1982), $C P^{*} N H$ in Negroids (Shokier and Shreffler, 1970; Mohrenweiser and Decker, 1982), CP*Michigan in Caucasoids and Negroids (Mohrenweiser and Decker, 1982) and $C P^{*} C$ in Mongoloids (Bajatzadeh and Walter, 1969).

In an earlier study of 4,026 Japanese adults residing in Hiroshima and Nagasaki, we detected one case each of $\mathrm{CP} \mathrm{C}_{\mathrm{NG} 1}$ and $\mathrm{CP} \mathrm{A}_{\mathrm{NG} 1}$ (Ferrell et al., 1977). In the present study, we extend this series, reporting on the findings in 22,367 children born to A-bomb survivors in Hiroshima and Nagasaki, as a part of a study whose purposes were described in detail in the first report of this series (Satoh et al, 1984).

\section{MATERIALS AND METHODS}

Population, sample preparation, family study, and nomenclature of variants. The general procedures employed in our study were described in detail in the earlier paper (Satoh et al., 1984). Briefly, blood samples were obtained from a total of 22,367 children (Hiroshima, 11,996; Nagasaki, 10,371) including those born to proximally exposed survivors (one or both parents exposed to A-bomb within $2,000 \mathrm{~m}$ from the hypocenter) and those born to distally exposed survivors (one or both parents exposed to A-bomb more than $2,500 \mathrm{~m}$ from the hypocenter). So far, no measurable genetic effect due to A-bomb exposure of the parents has been observed (Satoh et al., 1984). Therefore, in this report, data obtained from the children of the both groups are combined. About $30 \%$ of this sample consisted of siblings. When only one child was selected from one family using the same method described in the first report of this series, the number of unrelated children accounted for 14,964 (8,548 for Hiroshima, 6,416 for Nagasaki). The frequency of alleles which code variant types was calculated for this latter population. Blood samples were collected using ammonium-potassium oxalate or ACD solution as anticoagulants, centrifuged at $1,500 \times g$, and the plasma stored at $-70^{\circ} \mathrm{C}$ or in liquid nitrogen. Variants have been named by mobility class (A, fast; C, slow) and place (HR or NG) and sequence (1.2...) of discovery.

First screening and comparison of variants. As the first screening, vertical starch gel electrophoresis, an improvement of the method of Weitkamp et al. (1972), was conducted at $4^{\circ} \mathrm{C}$ using $0.0285 \mathrm{M}$ boric acid-LiOH, $\mathrm{pH} 8.05$, as bridge buffer and 
$0.061 \mathrm{~m}$ tris-citric acid and $0.0285 \mathrm{~m}$ boric acid-LiOH, pH 8.6, as gel buffer. Polyacrylamide slab gel electrophoresis (PAGE) was employed for confirmation and comparison of variants. PAGE was performed at $4^{\circ} \mathrm{C}$ with a gel composed of running gel $(\mathrm{T}, 7.2 \% ; \mathrm{C}, 2.8 \%)$ and stacking gel $(\mathrm{T}, 4.0 \% ; \mathrm{C}, 5 \%)$ and tank-gel buffer of $0.04 \mathrm{M}$ tris-glycine, $\mathrm{pH} 8.5$, employing the Pharmacia Mark II electrophoresis apparatus. Staining was conducted on the basis of oxidase activity of $\mathrm{CP}$ using dianisidine as a substrate following the method of Shreffler et al. (1967).

Tests on storage effects on migration rate. Samples were stored at $4{ }^{\circ} \mathrm{C}$ and $-20^{\circ} \mathrm{C}$, and one, two, three, and four weeks thereafter, PAGE was carried out. After dianisidine staining, the gel was examined to measure the concentration ratio of each band, using a densitometer. A second identically prepared gel underwent immunofixation as follows: 1) A cellulose acetate membrane was immersed in a solution containing an anticeruloplasmin antibody (Behring Inst.) diluted to $1 / 6$ by $0.15 \% \mathrm{NaCl}$, and this membrane was put on the gel and allowed to react for five hours at room temperature. 2) Bands of precipitation with Coomassie blue staining were identified. Protein quantitation of $\mathrm{CP}$ was carried out by single radial immunodiffusion (SRID). As controls, dithiothreitol was added to the samples to make the final concentration $0.1 \mathrm{~mm}$, incubated at $4^{\circ} \mathrm{C}$ for $30 \mathrm{~min}$, stored at $4^{\circ} \mathrm{C}$ or $-20^{\circ} \mathrm{C}$, and electrophoresis was done one, two, three, and four weeks thereafter.

\section{RESULTS}

Five rare variants were detected among a total of 22,367 children in addition to the two variants reported by Ferrell et al. (1977). The order of the migration rate of these variants including $C P A_{N G 1}$ and $C P C_{N G 1}$ and normal type $B$ is as follows (Fig. 1): $\mathrm{C}_{\mathrm{HR} 1}<\mathrm{C}_{\mathrm{HR} 2}<\mathrm{C}_{\mathrm{NG} 1}<\mathrm{B}<\mathrm{A}_{\mathrm{NG} 2}<\mathrm{A}_{\mathrm{HR} 1}<\mathrm{A}_{\mathrm{HR} 2}<\mathrm{A}_{\mathrm{NG} 1}$. Except for five cases of the homozygous phenotype $C P C_{N G 1}$, all the other variants encountered were heterozygous for the normal allele, $C P^{*} B$, and a variant allele. Table 1 shows the frequencies of each of these $C P$ phenotypes and alleles by city for the populations composed only of unrelated children. The frequency of $C P^{*} C N G 1$ was 0.0095 in Hiroshima and 0.0087 in Nagasaki, each being almost in polymorphic proportions. The allelic frequency of all of the other six variants was less than 0.001 , with no significant difference between the cities. $C P^{*} A H R 2$ and $C P^{*} C H R 2$ were detected in only one family. As a result of family studies, the five new types were all confimed as genetic variants (Table 2).

When plasma samples containing a $\mathrm{CP}$ variant were stored under various conditions, the electrophoretic pattern changed over time in some cases. Figure 2 shows the result of PAGE of plasma samples containing homozygous $\mathrm{CP}_{\mathrm{NG}} \mathrm{C}_{\mathrm{N}}$ wich had been stored at $4^{\circ} \mathrm{C}$ for four weeks. After two weeks of storage, in addition to the $\mathrm{CP} \mathrm{C}_{\mathrm{NG} 1}$ band, a new band, both dianisidine and immunofixation positive, appeared anodal to normal $\mathrm{B}$ band. At the same time, the staining intensity of $\mathrm{CP}_{\mathrm{NG}}$ decreased almost to the same level as that of the new band. With further elapse 


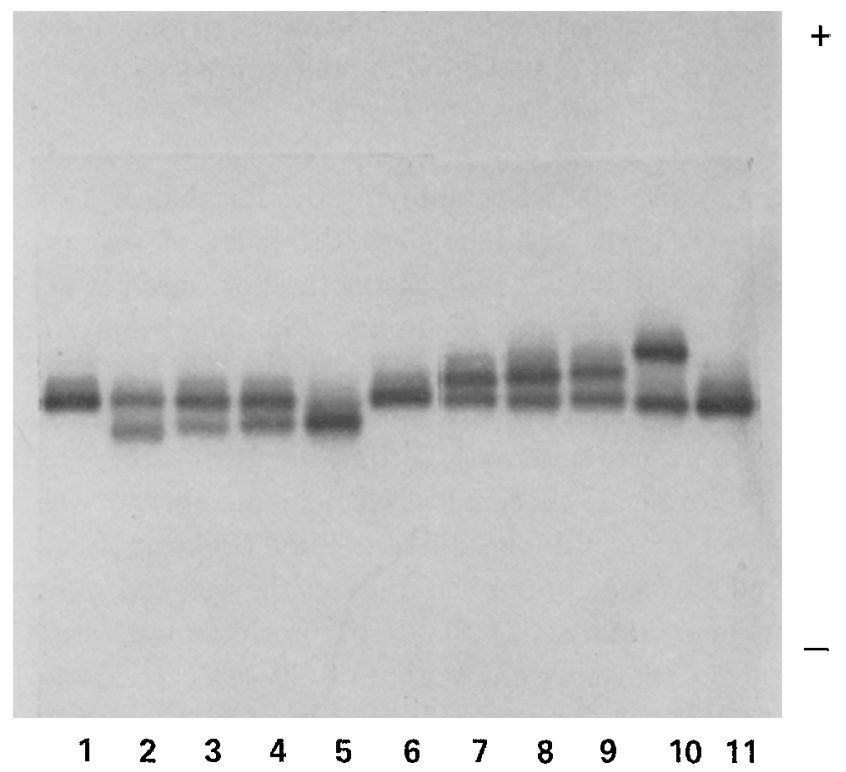

Fig. 1. Ceruloplasmin variants encountered in the present study on polyacrylamide slab gel electrophoresis. 1, 6 and 11, CP B; 2, CP B/CHR1; 3, CP B/CHR2; 4, CP B/ CNG1; 5, CP CNG1; 7, CP B/ANG2; 8, CP B/AHR1; 9, CP B/AHR2; 10, CP B/ANG1.

Table 1. Ceruloplasmin phenotypes observed among children of Hiroshima (HR) and Nagasaki (NG) and allele frequencies among the unrelated children.

\begin{tabular}{|c|c|c|c|c|c|c|c|c|}
\hline \multirow{2}{*}{ Phenotype } & \multirow{2}{*}{ Total } & \multicolumn{3}{|c|}{ Unrelated children ${ }^{2}$} & \multirow{2}{*}{ Allele } & \multirow{2}{*}{ Combined } & \multirow{2}{*}{ HR } & \multirow{2}{*}{ NG } \\
\hline & & Combined & $\mathrm{HR}$ & $N G$ & & & & \\
\hline $\mathrm{CP} \mathbf{B}$ & 21,917 & 14,678 & 8,380 & 6,298 & $C P * B$ & .99034 & .9901 & .9907 \\
\hline CP B/ANG1 & 16 & 10 & 3 & 7 & $C P^{*} A N G I$ & .00033 & .0002 & .0006 \\
\hline CP B/ANG2 & 2 & 1 & 0 & 1 & $C P^{*} A N G 2$ & .00003 & 0 & .0001 \\
\hline CP B/AHR1 & 2 & 2 & 2 & 0 & $C P^{*} A H R I$ & .00007 & .0001 & 0 \\
\hline CP B/AHR2 & 2 & $0 \mathrm{~b}$ & 0 & 0 & $C P^{*} A H R 2$ & $0^{b}$ & 0 & 0 \\
\hline $\mathrm{CP} \mathrm{B} / \mathrm{C}_{\mathrm{NG} 1}$ & 419 & 268 & 159 & 109 & & & & \\
\hline CP CNG1 & 5 & 3 & 2 & 1 & $C P^{*} C N G l$ & .00916 & .0095 & .0087 \\
\hline CP B/CHR1 & 3 & 1 & 1 & 0 & $C P * C H R I$ & .00003 & .0001 & 0 \\
\hline $\mathrm{CP} \mathrm{B} / \mathrm{CHR} 2$ & 1 & 1 & 1 & 0 & $C P^{*} C H R 2$ & .00003 & .0001 & 0 \\
\hline Total & 22,367 & 14,964 & 8,548 & 6,416 & & & & \\
\hline
\end{tabular}

a The population of unrelated children was composed of children without siblings and those who received the first test among their siblings. $b$ According to the above described rule, the brother with normal phenotype (CP B) who fulfilled the condition was selected as a member of the population and the propositus having variant phenotype was excluded. 
Table 2. Family studies of new ceruloplasmin variants encountered in this study.

\begin{tabular}{|c|c|c|c|c|}
\hline City & Propositus & Mother & Father & Siblings \\
\hline 1. NG & (1) $B / A_{N G 2}$ & 0 & 0 & \\
\hline 2. HR & (1) $B / A_{N G 2}$ & 0 & $\square$ & \\
\hline 3. $\mathrm{HR}$ & (1) $B / A_{H R 1}$ & (1) & $\square$ & $\bigcirc$ \\
\hline 4. $\mathrm{HR}$ & (1) $B / A_{H R 1}$ & NT & NT & \\
\hline 5. HR & D $\mathrm{B} / \mathrm{A}_{\mathrm{HR2}}$ & ( & $\square$ & $O \oplus 0$ \\
\hline 6. HR & $\mathbb{B} / \mathrm{C}_{\mathrm{HR1}}$ & 0 & D & 0 \\
\hline 7. $\mathrm{HR}$ & $\mathrm{B} / \mathrm{C}_{\mathrm{HR} 1}$ & (1) & $\square$ & \\
\hline 8. HR & $\bar{B} / \mathrm{C}_{\mathrm{HR2}}$ & 0 & $\square$ & \\
\hline
\end{tabular}

O , Heterozygote; $\bigcirc \square$, Normal; $†$, Deceased; NT, Not tested

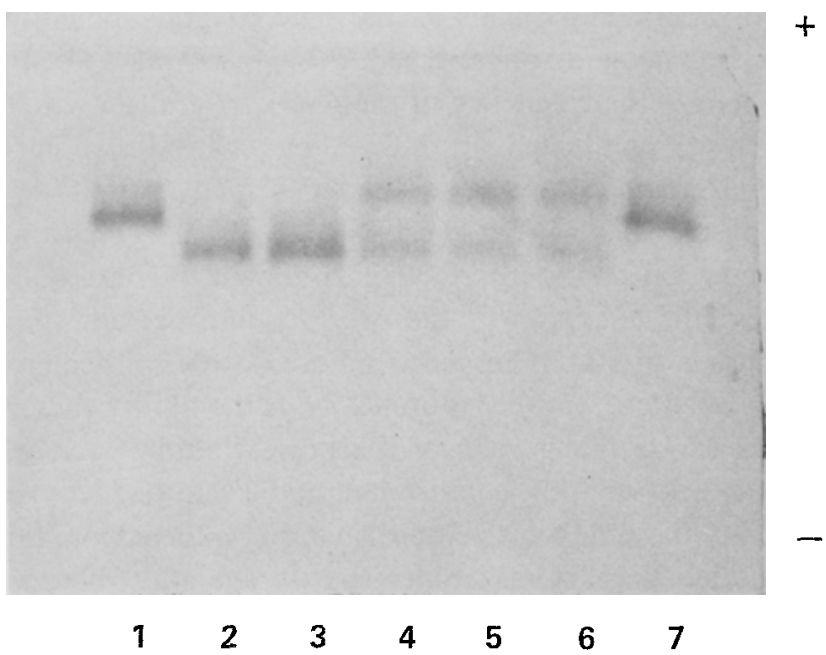

Fig. 2. Storage effects at $4^{\circ} \mathrm{C}$ on a ceruloplasmin variant (CP CNG1) shown by polyacrylamide slab gel electrophoresis. 1 and 7 , control fresh $C P B ; 2$, fresh $C_{P} C_{N G 1}$; 3, CP $\mathrm{C}_{\mathrm{NG} 1}$ after 1 week; 4, CP $\mathrm{C}_{\mathrm{NG1}}$ after 2 weeks; 5, CP $\mathrm{C}_{\mathrm{NG}}$ after 3 weeks; 6 , CP CNG1 after 4 weeks.

in time, the staining intensity of the new band increased, and two weeks thereafter, the pattern was similar to phenotype $\mathrm{CP} A / \mathrm{C}_{\mathrm{NG}}$. As for heterozygous $\mathrm{CP} B / \mathrm{C}_{\mathrm{NG}}$, the staining intensity of the $\mathrm{CP} B$ band was always stronger than the $C P C_{N G 1}$ band. The difference in staining intensity of the two bands became greater with time, and there or four weeks later, $\mathrm{CP} \mathrm{C}_{\mathrm{NG} 1}$ band became undetectable by either dianisidine staining or immunofixation. On the other hand, the width of the CP B band became broader on the anodal side, but the pattern was scored as phenotype CP B.

In the electrophoretic pattern of heterozygous $\mathrm{CP} B / \mathrm{A}_{\mathrm{NG}}$, the staining intensity 
of the CP $A_{N G 1}$ band was slightly stronger than that of CP B band no change of migration rate with time was observed. The band pattern of homozygous CP B also showed no change over time. At the same time, the protein concentration of $\mathrm{CP} \mathrm{C}_{\mathrm{NG} 1}, \mathrm{CP} \mathrm{B} / \mathrm{C}_{\mathrm{NG} 1}, \mathrm{CP} \mathrm{B} / \mathrm{A}_{\mathrm{NG} 1}$, and $\mathrm{CP} \mathrm{B}$ quantitated by SRID showed no change over time. When samples were stored at $-20^{\circ} \mathrm{C}$, change of migration rate was not apparent four weeks later, and those stored in liquid nitrogen showed no change in migration rate for at least six years. Storage of samples at $4^{\circ} \mathrm{C}$ after dithiothreitol processing prevented a part (not all) of storage effects on the migration rate of the $\mathrm{CP} \mathrm{C}_{\mathrm{NG}}$ band.

\section{DISCUSSION}

The variant designated as $\mathrm{CP} \mathrm{C}_{\mathrm{NG} 1}$ which migrates cathodal to normal $\mathrm{CP} B$ band was identified in 268 individuals as the heterozygous phenotype $\mathrm{CP} B / \mathrm{C}_{\mathrm{NG}}$ and in three as the homozygous phenotype CP $\mathrm{C}_{\mathrm{NG} 1}$ among 14,964 unrelated children studied. Therefore, the frequency of the $C P^{*} C N G 1$ allele was 0.00916 . However, our previous study conducted on 4,026 subjects in Hiroshima and Nagasaki, the same cities of the present study, which included many parents of these children, identified only one case of this variant (Ferrell et al., 1977). This apparent disparity between the two investigations merits further comment. There seems to be essentially no difference in the character of the previous and present study populations. Furthermore, conditions of starch gel electrophoresis were basically the same. We have experienced a difference between starch lots in the ability to separate CP variants. In the starch gel electrophoresis of the present study, even when separation of variants was poor, confirmation could be made by PAGE. A second important factor is judged to be the effect of freeze-thawing conducted on samples used for the confirmation studies of variants. Since the storage effect observed in $\mathrm{CP} \mathrm{C}_{\mathrm{NG} 1}$ (Fig. 2) became apparent after freeze-thawing, it is thought that, of the two bands of the heterozygote, $\mathrm{CP} \mathrm{C}_{\mathrm{NG} 1}$ band showed remarkable decrease of staining intensity as well as its change of migration rate, it could be mistaken for the CP B. In the detection of CP variants, full consideration should be given to the selection of the supporting gel medium, the storage, and treatment of samples.

The polymorphic allele of $\mathrm{CP}, C P^{*} A$ was observed in American and African Negroid populations in a frequency of $0.041-0.147$ and in Caucasoid populations (Greeks, Germans, and Irelanders) in a frequency of 0.01-0.068 (Kellermann and Walter, 1972). However, Morhenweiser and Decker (1982), in a detailed examination on 1,853 Caucasians and 199 Blacks in the US by PAGE using a combination of three types of buffers and gradient gel, reported that the Negroid population had two types of variants with migration rate faster than CP B, CP A and CP Michigan, with the allele coding for each variant being in polymorphic frequencies of 0.033 and 0.010 . In the Caucasoid population, the CP A phenotype which was detected in Blacks was not identified, and only CP Michigan was observed, the allele 
frequency being 0.012 . They suggested that as the frequency of the CP Michigan was quite similar to that reported for $C P^{*} A$ in European Caucasian populations by Kellermann and Walter (1972), the CP A variant which had been previously reported in the Caucasoid population was actually CP Michigan. Its migration rate is slightly slower (more cathodal) than that of CP A, the variant of Blacks. CP Michigan was thought to be the more ancient of the two because it was common to both populations.

Fast migrating variants present in polymorphic frequencies have not been identified in Japanese and Korean populations. On the other hand, in addition to CP $\mathrm{C}_{\mathrm{NG1}}$ reported here, the $\mathrm{CP} \mathrm{C}$ variant was reported as polymorphic in a Korean population (Bajatzadeh and Walter, 1969). They examined 115 Koreans using horizontal starch gel electrophoresis and detected one homozygote $\mathrm{CP} \mathrm{C}$ and one heterozygote $\mathrm{CP} \mathrm{B} / \mathrm{C}$. The frequency of the $C P^{*} C$ allele was thus 0.013 . Unfortunately we have not had the opportunity to compare $C P \mathrm{C}_{\mathrm{NG1}}$ and the Korean $\mathrm{CP}$ $\mathrm{C}$ variant on the same gel. However, it seems possible that these variants may be identical. If this is so, as is the case with TF $D_{\text {chi }}$, these CP variants may be useful markers for Mongoloid populations. Therefore, identification of these variants is of more than routine interest.

We already reported that on PAGE when the $C P A_{N G 1}$ and $C P C_{N G 1}$ variants detected in our population were compared with the $\mathrm{CP} A$ and $\mathrm{CP} C$ variants detected in a Negroid population, the order of the migration rates was CP $A_{N G 1}>C P A$ and $\mathrm{CP} \mathrm{C}_{\mathrm{NG} 1}>\mathrm{CP}$ C (Ferrell et al., 1977). However, comparison between the newly detected five variants and those detected in other populations was not possible. Since CP is a large molecule composed of 1,046 amino acids (Takahashi et al., 1984), the probability that electrophoretically identical rare variants detected in only two or three families result from the same type of amino acid substitution at the same site appears to be quite small.

Acknowledgment We are deeply indebted to Dr. J.V. Neel for invaluable support.

\section{REFERENCES}

Bajatzadeh, M. and Walter, H. 1969. Studies on the population genetics of the ceruloplasmin polymorphism. Humangenetik 8: 134-136.

Ferrell, R.E., Ueda, N., Satoh, C., Tanis, R.J., Neel, J.V., Hamilton, H.B., Inamizu, T., and Baba, K. 1977. The frequency in Japanese of genetic variants of 22 proteins. I. Albumin, ceruloplasmin, haptoglobin transferrin. Ann. Hum. Genet. 40: 407-418.

Kellermann, G. and Walter, H. 1972. On the population genetics of the ceruloplasmin polymorphism. Humangenetik 15: 84-86.

McAlister, R., Martin, G.M., and Benditt, E.P. 1961. Evidence for multiple ceruloplasmin components in human serum. Nature 190: 927-929.

Mohrenweiser, H.W. and Decker, R.S. 1982. Identification of several electrophoretic variants of human ceruloplasmin including CpMichigan, a new polymorphism. Hum. Hered. 32: 369373. 
Neel, J.V., Tanis, R.J., Migliazza, E.C., Spielman, R.S., Salzano, F.M., Oliver, W.J., Morrow, M., and Bachofer, S. 1977. Genetic studies of the Macushi and Wapishana Indians. I. Rare genetic variants and a "private polymorphism" of esterase A. Hum. Genet. 36: 81-107.

Satoh, C., Takahashi, N., Asakawa, J., Masunari, N., Fujita, M., Goriki, K., Hazama, R., and Iwamoto, K. 1984. Electrophoretic variants of blood proteins in Japanese. I. Phosphoglucomutase-2. Jpn. J. Hum. Genet. 29: 89-104.

Shokeir, M.H.K. and Shreffler, D.C. 1970. Two new ceruloplasmin variants in Negroes-data on three populations. Biochem. Genet. 4: 517-528.

Shreffler, D.C., Brewer, G.J., Gall, J.C., and Honeyman, M.S. 1967. Electrophoretic variation in human serum ceruloplasmin: A new genetic polymorphism. Biochem. Genet. 1: 101-115.

Takahashi, N., Ortel, T.L., and Putnam, F.W. 1984. Single-chain structure of human ceruloplasmin: The complete amino acid sequence of the whole molecule. Proc. Natl. Acad. Sci. USA 81: 390-394.

Tanis, R.J., Neel, J.V., Dovey, H., and Marrow, M. 1973. The genetic structure of a tribal population, the Yanomama Indians. IX. Gene frequencies for 18 serum protein and erythrocyte enzyme systems in Yanomama and five neighboring tribes; nine new variants. Am. J. Hum. Genet. 25: 655-676.

Weitkamp, L.R., Arends, T., Gallango, M.L., Neel, J.V., Schultz, J., and Shreffler, D.C. 1972. The genetic structure of a tribal population, Yanomama Indians. III. Seven serum protein systems. Ann. Hum. Genet. 35: 271-279. 